Jean-Claude CHEVALIER

Paris

\title{
Cesar Chesneau du Marsais et Maurice Gross. DEUX RÉVOLUTIONS ÉPISTÉMOLOGIQUES EN MIROIR.
}

La Linguistique cartésienne de Chomsky a centré la réflexion des linguistes contemporains sur la Grammaire générale de Port-Royal : la généralité des règles, leur petit nombre, leur vertu explicative et constructive permettaient de jeter un pont de Descartes à Humboldt; et la plupart des générativistes s'en contentaient. Très tôt pourtant, Maurice Gross, dont on sait avec quel œil critique il regardait les simplifications du chomskysme, s'était intéressé à relire Du Marsais, au double travail d'analyse qu'exerçait sur la langue ce philosophe-pédagogue à partir des années 1730. D'une part, un travail de grammairien et de philosophe qui ferait de lui le grammairien de l'Encyclopédie dans sa première partie (jusqu'à l'article «Grammaire ») et l'auteur d'innovations remarquables, d'autre part un travail de sémanticien et de rhéteur qui le conduirait à publier à partir de 1730 son livre Des Tropes. Des Tropes articulait une double approche : d'une part, une approche méthodique des variables de la parole, constitutives du discours qui se fait, d'autre part, et conjointement, une description raisonnée de la constitution du système de la langue.

Variables de la parole dont les figures sont l'élément le plus marquant. Bien loin que ces figures soient le lieu de l'artifice, comme le voudraient certains, Du Marsais les tient pour éléments constituants du discours, issus des sensations et sentiments. Et Du Marsais cite Bretteville : « J'ai pris souvent plaisir à entendre des paysans s'entretenir avec des figures de discours si variées, si vives, si éloignées du vulgaire, que j'avais honte d'avoir si longtemps étudié l'éloquence, voyant en eux une certaine rhétorique de nature beaucoup plus persuasive et plus éloquente que toutes nos rhétoriques artificielles. » Et il commente : « Il n'y a rien de si aisé et de si naturel » (Tropes, 18).

La science du langage enseigne une rhétorique artificielle, la nature une rhétorique sans artifice. Distinction qu'avait déjà notée un siècle plus tôt Malherbe en évoquant la richesse de figures dont débordent les parlers de la Place Maubert et des crocheteurs du Port-aux-Foins. Mais le point de vue a changé. Malherbe ne pensait que distinctions sociales, Du Marsais se réfère à la nature. Locke est passé par là ; et aussi son lecteur et interprète, le Père Buffier, comme intermédiaire essentiel.

Car ce jésuite, adepte de la force tranquille, a su imposer au public universitaire un modèle né d'une alliance nouvelle entre les principes de Descartes et les innovations d'un Locke récemment traduit par Coste. Exalter la raison dans la langue, certes; mais pour le lecteur de Locke qu'il est, les bâtiments de la langue et de l'expression ne sont pas préétablis, ils se forment dans le jeu et le développement de sensations s'inscrivant dans un terrain prédisposé, dans le cadre de la dialectique de la perception et de la formalisation; jeu parfois désordonné qui peut déborder, contredire même les principes de la raison sur lesquels était fondée la démarche de Port-Royal, centrée sur une unité logico-linguistique : la proposition. En conséquence, Buffier organise les éléments du discours dans des 
regroupements anciens certes, mais répondant à une définition nouvelle stricte : la phrase, dont il est l'initiateur en syntaxe du discours. La phrase est tenue pour une unité formelle que Buffier adopte comme unité grammaticalo-discursive, fondée sur les découpages de sens de l'énonciation. On peut, à bon droit, parler d'une révolution de l'épistémè.

Ce que reprend Du Marsais une vingtaine d'années plus tard quand il propose un petit livre des Tropes qui aura un éclatant succès. Et qui s'ouvre par un paradoxe :

«Bien loin que les figures s'éloignent du langage ordinaire des hommes, ce seraient au contraire les façons de parler sans figures qui s'en éloigneraient, s'il était possible de faire un discours où il n'y eût que des expressions non figurées. » (p. 19)

C'est donc le langage sans figure qui est l'artifice aussi bien que l'excès de figures. Pour développer sa pensée, il recourt à une métaphore :

«Imaginez-vous pour un moment une multitude de soldats, dont les uns n'ont que l'habit ordinaire qu'ils avaient avant leur engagement, et les autres ont l'habit uniforme de leur régiment. Ceux-ci ont tous un habit qui les distingue, et qui fait connaître de quel régiment ils sont : les uns sont habillés de rouge, les autres de bleu, de blanc, de jaune, etc. Il en est de même des assemblages de mots qui composent le discours; un lecteur instruit rapporte un tel mot, une telle phrase à une telle espèce de figure, selon qu'il y reconnait la forme, le signe, le caractère de cette figure. Les phrases et les mots qui n'ont la marque d'aucune figure particulière, sont comme les soldats qui n'ont l'habit d'aucun régiment ; elles n'ont d'autre modification que celles qui sont nécessaires pour faire connaître ce qu'on pense. » (p. 25-26)

Enumérer les figures, c'est définir les éléments constituants du discours. Du Marsais en inventorie treize qu'il emprunte à la tradition, résultant « de la nécessité ou de l'ornement », à savoir: catachrèse, onomatopée, métonymie, synecdoque, antonomase, ironie, antiphrase, périphrase, énigme, hyperbole, litote, métaphore, allégorie.

Après lui, Beauzée en restreindra le nombre à trois : métaphore, métonymie et synecdoque. Reprenant les trois relations de Locke (contiguïté, comparaison, abstraction) et annonçant la célèbre base d'analyse de Roman Jakobson. Dans l'ordre : rapports de similitude, de correspondance et de connexion; relations fondamentales donc du jeu entre les idées.

Les éléments constituants ainsi définis, on peut en envisager la construction, définir une syntaxe des éléments de relations. Ce qu'il fait au chapitre intitulé : « Du discours considéré grammaticalement et des parties qui le composent»; chapitre considéré comme un aboutissement, un au-delà du discours. Parti de la périphérie, le grammairien analyse le centre ; et il pourra ainsi aborder les textes artificiels dépouillés de toute figure aussi bien que les textes figuratifs, l'unité de découpage demeurant la phrase. Opération ainsi définie :

«Dans toutes ces phrases, il y a toujours le signe de l'activité de l'esprit qui applique, qui rapporte, qui adapte une perception ou une qualification à un objet; mais qui l'adapte ou avec la forme du commandement, ou avec celle de condition, de souhait, de dépendance ; mais il n'y a point là de décision qui affirme ou qui nie relativement à l'état positif de l'objet. 
Voilà une différence essentielle entre les propositions : les unes sont directement affirmatives ou négatives, et énoncent des jugements; les autres n'entrent dans le discours que pour y énoncer certaines vues de l'esprit. » (p. 98)

Système souple permettant de répondre à une double postulation, intellectuelle et émotive.

Cette division d'ensemble une fois notée, Du Marsais propose que l'analyste distingue le rapport d'identité, par exemple entre le nom et l'adjectif ou entre le verbe et le sujet et le rapport de détermination, ainsi des expressions introduites par de comme « la lumière du soleil» ou «Tu aimeras le Seigneur ». Plus tard, Du Marsais coiffera cette dénomination logique d'un autre terme, le terme de «complément»; que Beauzée popularisera parce qu'il permet une plus grande généralisation; et donc une adaptation plus satisfaisante aux réalisations particulières du discours. La souplesse de ce dispositif permettra d'aborder aussi bien des segments élémentaires que des textes poétiques chargés de multiples effets, mais dépendants des mêmes principes, car, écrit-il, « Les principes métaphysiques de la construction sont les mêmes dans toutes les langues » (p. 159) Ce qui lui permettra de proposer une analyse provocatrice de la célèbre (à l'époque) Bergerie de Mme Deshoulières :

\section{Hélas! petits moutons, que vous êtes heureux \\ Vous paissez dans nos champs, sans souci, sans alarmes \\ Relisez la suite et les commentaires de notre grammairien-philosophe (p. 159).}

Résumons: l'ambitieuse machinerie de Du Marsais vise à recouvrir le fonctionnement du discours, à partir des variables que sont les tropes et les figures de discours, lesquelles sont la nature même de la parole, et à définir la systématique des éléments de relation qui supportent le système sous-jacent et lui permettent de fonctionner en langue.

On retrouvera le même système d'ensemble chez Maurice Gross, mais, en quelque sorte, inversé. Le jeune polytechnicien, en 1961, est affecté comme boursier au Labo de Chomsky, au MIT. Il est chargé par son directeur au CNRS, Sestier, d'examiner l'apport de la nouvelle linguistique pour la traduction automatique. Même si, quelques mois plus tard, comme les Américains (le célèbre rapport de Bar-Hillel!), Sestier abandonne les ambitions de la traduction automatique, la recherche de la formalisation de tous les éléments de langue restera dominante chez le jeune chercheur, renforcée par la fréquentation de Zellig Harris dont Gross devient l'assistant à l'Université de Pensylvanie en octobre 1964, une formalisation qui prétend rendre compte des exceptions mêmes et se marquera dans les publications d'après 1967. Néanmoins le classement des structures par tables, réunissant des verbes de même construction, dotés de propriétés comparables, fait apparaître que ces tables généralisantes sont débordées par les spécificités de la parole. C'est ce qui ressort de la compilation de Méthodes en syntaxe (1975). Tout l'effort de M. Gross et de son équipe consistera alors à s'orienter vers une confrontation par tableaux informatiques entre l'inventaire des tables généralisantes et les réalisations particulières. 
Le premier effort - et le plus important - visera à définir dans le cadre des Méthodes des opérations de transfert entre structures. On se contentera d'en énumérer quelques-unes empruntées à un article bilan de 1981, examinant la récursivité des structures (M. Gross, «Les bases empiriques de la notion de prédicat sémantique », Langages 63 ).

Un point fondamental repose sur les possibilités de transmutation entre nom et verbe ; ainsi des phrases :

«Max favorise Luc. Max se bagarre avec Luc. »

Elles peuvent être tenues pour avoir des expressions synonymiques :

« Max donne un avantage à Luc. Max mène une bagarre avec Luc. » Etc., etc.

Il s'agit là d'une transformation particulièrement productive et efficace qui implique l'analyse de ce que Gross appelle des «verbes supports », élément fondamental des transferts de type verbo-nominal. Un autre type de transfert, apparenté et très efficace lui aussi, a été dénommé « restructuration ». Il s'agit d'une règle qui place un complément de nom en position de verbe, comme par exemple dans les équivalences suivantes :

«Max amuse Eva par ses minauderies

= Les minauderies de Max amusent Eva

= Les minauderies que fait Max amusent Eva. » (p. 19)

Ces restructurations observables chez le parleur permettent une très grande souplesse discursive et donc des mises en valeur différente. La découverte des transformations dans les structures de base conduit le parleur à l'invention d'éléments combinables pour la construction de discours.

Certes ces transformations impliquent de légères différences de sens, et c'est bien la raison pour laquelle elles sont utilisées avec souplesse dans le discours ordinaire. Ainsi de cet exemple ; allégué par M. Gross et fondé sur le jeu des déterminants :

« Max mange des kilos de pain / Max mange le pain par kilos. »

Intuitivement, ces deux variables plus ou moins synonymes ne sont pas exactement équivalentes, la seconde dénonçant un excès. L'introduction de C'est...qui, C'est...que confirmera cette intuition. L'analyse d'une équivalence de même genre («Max apprécie le travail de Luc» / «Max apprécie Luc pour son travail ») montre que l'interversion introduit une variable psychologique qui s'insérera dans l'efficacité d'un discours. Ces manipulations introduisent des jeux de variables, inconnues des analyses ordinaires des grammairiens, mais qui attestent la capacité pour le parleur entraîné de disposer d'une capacité à créer des variables sans fin. Incessamment, le centre nourrit sa périphérie.

L'inventaire des verbes opérateurs prouve la démultiplication constante des possibilités dans ce type de construction. Ainsi le recours à faire permet, comme 
César Chesneau Du Marsais et Maurice Gross. Deux révolutions épistémologiques en miroir.

l'avait déjà remarqué Lucien Tesnière dans sa Syntaxe structurale, d'ajouter un agent aux relations engagées :

«Max le fait dormir » / « Max lui en fait boire ».

Par extension, on peut relever d'autres paires qui permettent d'ajouter un argument supplémentaire :

« Max est dans le désarroi » / « Cet accident a plongé Max dans le désarroi ».

Cette intervention de l'opérateur n'introduit qu'un léger changement, mais très précieux : il permet de préciser l'élément causatif. On peut ainsi continuer les recherches d'éléments opérateurs, ce qui conduit à inventorier de légers changements de sens. Ainsi, à partir de la transformation de «L'argent influe sur Max » en «L'argent a de l'influence sur Max », on pourra tirer la suite :

«L'argent prend de l'influence» / «perd de l'influence » / «garde de l'influence sur Max. ». On dirait presque «à l'infini ».

Le linguiste insérera l'inférence de ce type de séries dont le rôle dans la construction de multiples variations de discours est essentielle. Multiples variations découlant du principe cher à Gross : «Les entrées du lexique ne sont pas des mots, mais des phrases » (48) : ce qui conduit à ne pas séparer dans l'énonciation les noms des phrases. Et à observer scrupuleusement le principe qu'on raisonne - et qu'on parle - avec des ensembles de mots déjà à l'œuvre et non des suites de symboles sous-jacents comme dans la grammaire générative. On ne quitte pas le fil de la constitution du discours.

Car les transformations du discours sont ici à l'œuvre. Point important : le jeu des transformations est de même type dans les constructions libres et dans les expressions semi-figées (et, dans une mesure évidemment très limitée, figées). Ce qui explique la commodité de leur emploi et leur inscription dans la construction libre.

Chemin faisant, les équipes de M. Gross n'ont pas pu ignorer deux formes particulières de ces ensembles lexicaux : les proverbes et les lexies, c.-à-d. des ensembles lexicaux partiellement solidifiés, parfois totalement. Quand on a la patience d'en faire l'inventaire, on s'aperçoit que ces deux ensembles tiennent une part importante dans la construction de notre parole, employés par milliers ; on les relève plus ou moins incidemment dans les dictionnaires, mais très rarement dans les grammaires. C'est dans ce double domaine que M. Gross a lancé des équipes internationales; et on s'aperçoit alors rapidement que c'est un phénomène universel.

$1^{\circ}$ Les proverbes. Ils sont marqués socialement comme signe d'une pensée primitive. Certes, ils jouent un grand rôle dans l'apprentissage des langues jusqu'au $16^{\text {ème }}$ siècle inclus. Les recueils de synonymes proverbiaux sont un des modes d'apprentissage dans les manuels de la Renaissance. Mais on les retrouve aussi dans les Lettres, qu'on pense aux aphorismes de Sancho Pança arrachés à ce grotesque ou aux propos du même genre dont Molière gratifie ses personnages 
ridicules. La condamnation de l'emploi systématique des proverbes intervient sans doute avec le rationalisme de l'âge classique qui privilégie les démarches analytiques. Un discours préalablement construit est condamné par les beaux esprits; la démarche de discours doit suivre l'analyse généralement propositionnelle.

Même remarques en ce qui concerne les dictionnaires. Le premier Dictionnaire de l'Académie française, l'édition de 1694, accorde encore aux proverbes une importance primordiale dans l'énonciation de vocables comme supports de construction. Les éditions suivantes restreindront ce privilège et priveront le parleur d'un moyen d'expression commode.

Ils n'en persistent pas moins, plus souterrainement peut-être. Dans les banlieues du langage, la chasse aux proverbes est ouverte. Les relevés effectués aujourd'hui au Québec, en Belgique, dans le Valais, en Italie attestent que ce réservoir de construction des discours recèle d'immenses ressources, même si les proverbes ne font partie que marginalement de la partie la plus accessible aux analyses linguistiques.

$2^{\circ}$ Même remarque pour l'inventaire des lexies ou expressions toutes faites. On en retrouvera des modèles dans de nombreuses compilations et, particulièrement, celles qui ont été élaborées dans les différents centres de recherche dépendant du Laboratoire de Maurice Gross et de Gaston Gross. L'un des plus remarquables me semble être l'inventaire établi par le plus jeune (en esprit ; il est né en 1920) de ses élèves, je nomme Jean Dubois qui a travaillé avec sa femme, l'excellente linguiste Françoise Dubois, spécialiste des études anglophones pour composer une extraordinaire compilation: Locutions en français, aujourd'hui consultable au Laboratoire informatique de Paris 10, le Modyco et pour l'édition papier (372 pages serrées) disponible chez l'auteur.

Partant d'ensembles de constituants, les Dubois investissent des variables relativement limitées; «les constituants sont indissociables dans les locutions » écrivent-ils. Ainsi dans «Paul lit entre les lignes» l'expression entière est figée, tandis que dans «On aperçoit à l'arrière-plan sa figure émaciée », seule l'expression prépositionnelle est figée. C'est que le figement est plus ou moins important, plus ou moins partiel ; par là ces locutions offrent au locuteur une plus ou moins grande souplesse, plus ou moins de place à l'improvisation. Le parleur est dans un lieu intermédiaire entre l'emprunt à des expressions toutes faites et la recréation par compétence grammaticale, bref à la frontière entre langue et parole. Ce que disent parfaitement les Dubois :

«Il est bien évident que les locutions retenues dans cet ouvrage ne représentent qu'un échantillon très partiel des GN, GV et GA locutionnels existants, répertoriés ou non dans les dictionnaires les plus élaborés ; ces locutions qui structurent syntaxiquement, sémantiquement, et sans doute idéologiquement tous les énoncés sont issues de modèles cumulatifs et toujours productifs; leur nombre ne peut donc que s'accroître. Sans doute n'était-il possible que de définir les modèles eux-mêmes ; sans doute est-on encore loin de compte. » (20)

Autre aspect de cette prise en compte de groupes modélisés. Les grammairiens générativistes pur jus comme Nicolas Ruwet, à la même époque, relèvent, pour 
appuyer leur argumentation, des données empruntées à la littérature et autres genres codifiés, signe d'une évolution qui tient le discours comme référent indispensable de la détermination des valeurs. Si l'on ajoute, pour finir, que, toujours à cette époque et un peu plus tard - les années 1990 -, c'est le moment même où, grâce à la découverte de nouveaux manuscrits de Saussure, dits « de l'Orangerie » par Simon Bouquet et Rudolf Engler, manuscrits qui soulignent le rôle de la parole, Saussure même est embrigadé dans cette révolution épistémologique, lieu de rencontre de différents courants d'interprétation. Tous éléments concourant à un mouvement d'ensemble significatif de cette fin de siècle.

\section{BIBLIOGRAPHIE SUCCINCTE}

\section{Pour Du Marsais :}

Chevalier, Jean-Claude (2006, éd. originale 1968, Droz), Histoire de la syntaxe : Naissance de la notion de complément dans la grammaire française (15301750). Paris, Champion.

Du Marsais. Euvres. Langlois, An VIII, tomes 1 et 2.

DOUAY-SOUBLIN Françoise (à paraître), Nouvel examen de la Grammaire raisonnée de Madame du Châtelet.

\section{Pour Maurice Gross :}

BALIBAR-MRABTI, Antoinette (à paraître), Noms propres, vulgarisation, changement.

Chevalier, Jean-Claude, ENCREve, Pierre (2006), Combats pour la linguistique, de Martinet à Julia Kristeva, ENS Lyon.

GARDENT, Claire et al., (2005), Le lexique-grammaire de M. Gross et le traitement automatique des langues, Nancy, Loria et Atilf.

Gross, Maurice (1975), Méthodes en Syntaxe, Paris, Hermann.

GROSS, Maurice (1981), Les bases empiriques de la notion de prédicat sémantique, Langages 63, pp. 7-52.

\section{RESÚMEN}

Maurice Gross conocía muy bien la historia de las gramáticas y poseía una estupenda biblioteca de manuales antiguos. Tenía una predilección por la gramática general y especialmente por Du Marsais que proponía a los lectores de la Enciclopedia de Diderot una doble teorización del lenguaje que se refiere al tema de este simposio. En el Tratado de los Tropos, Du Marsais hizo un inventario sobre las variables que constituyen las distintas figuras del discurso y en la Enciclopedia, propuso una teorización de las estructuras de la oración propia para constituir una gramática general. 